\title{
Image Overlay Guidance for Needle Insertion in CT Scanner
}

\author{
Gabor Fichtinger ${ }^{1}$, Anton Deguet ${ }^{1}$, Ken Masamune ${ }^{2}$, Emese Balogh ${ }^{1}$, Gregory Fischer ${ }^{1}$, Herve Mathieu ${ }^{1}$, \\ Russell H. Taylor ${ }^{1}$, S. James Zinreich ${ }^{1}$, and Laura M. Fayad ${ }^{1}$
}

\begin{abstract}
We present an image overlay system to aid needle insertion procedures in CT scanners. The device consists of a display and a semitransparent mirror that is mounted on the gantry. Looking at the patient through the mirror, the CT image appears to be floating inside the patient with correct size and position, thereby providing the physician with two-dimensional "X-ray vision" to guide needle insertions. The physician inserts the needle following the optimal path identified in the CT image rendered on the display and thus reflected in the mirror. The system promises to reduce $\mathrm{X}$-ray dose, patient discomfort, and procedure time by significantly reducing faulty insertion attempts. It may also increase needle placement accuracy. We report the design and implementation of the image overlay system followed by the results of phantom and cadaver experiments in several clinical applications.
\end{abstract}

\section{INTRODUCTION AND BACKGROUND}

$\mathbf{N}$ UMEROUS STUDIES have demonstrated the potential efficacy of computed tomography (CT) guided percutaneous needle-based interventions in a wide variety of medical problems. Contemporary practice, however, is limited by the freehand nature of needle placement, which requires mental registration of the patient's anatomy to the CT image in targeting and precise hand-eye coordination in inserting the needle. This frequently results in faulty needle placement attempts, especially in the case of a deep lesion adjacent to vital structures, which in turn increases the discomfort and radiation exposure of the patient, and lengthens the procedure. The recent appearance of real-time CT fluoroscopy has brought significant improvement to needle insertion procedures, but CT fluoroscopy is not without significant risks and shortcomings, it exposes patient and physician to real-time $\mathrm{X}$-ray dose and the physician's hand-eye coordination still remains a dominant factor. Presently no technological solution exists to assist freehand CT-guided needle placement in a simple, accurate, and inexpensive manner, without imparting additional radiation.

Practitioners generally agree that, given enough time and opportunity for intermittent CT imaging and adjustments, the target usually can be reached with appropriate accuracy. The important question is, however, whether the same objective could be achieved with just one attempt, because each correction requires taking an extra $\mathrm{CT}$ image (or even series of images) and reinsertion of the needle, which in turn increases the risk of post-procedure complication, discomfort, and radiation exposure to the patient. Therefore, eliminating

\footnotetext{
${ }^{1}$ Johns Hopkins University, Baltimore, MD, USA

${ }^{2}$ Tokyo Denki University, Japan
}

faulty needle placement attempts is a prime objective of our research.

A variety of tracked navigation systems and surgical robots [3], [14] have been investigated to assist image guided freehand insertions; all resulting in systems that are prohibitively complex and/or expensive for routine clinical use. Birkfellner et al. integrated computer graphics into the optical path of a head-mounted stereo binocular [1]. Sauer et al. reported another variant of head-mounted display (HMD) technology [12], in which two head-mounted cameras captured the real scene and a stereo HMD visualized the augmented scene. DiGioia et al. developed a volumetric image overlay system by projecting three-dimensional virtual image on a semitransparent mirror in which the physician simultaneously can observe the actual patient and computer-generated images [2], [4]. A similar device using this concept is commercially available under the brand name of Dexteroscope [7], which also involves elaborate preoperative calibration and requires realtime spatial tracking of all components, including the patient, physician's head, overlay display, mirror, and surgical tools. Grimson et al. graphically overlaid segmented 3D preoperative images onto video images of the patient [5]. This approach presented largely the same calibration and tracking problems as DiGioia's system, and in addition, the projected image also had to be warped to conform to the surface of the patient. Iseki et al. created a volumetric overlay display, and off-line registered to the patient in intracranial neurosurgery cases with the use of fiducials implanted into the skull [6], [11]. Current volumetric augmented reality devices require painstaking calibration and real-time spatial tracking. Tracked navigation systems have also known to have a plethora of problems and risk factors extensively characterized in the computer-aided surgery literature. Most current systems depend on complex and expensive hardware.

In the pursuit of simpler image overlay systems, our group [9], [10] and concurrently Stetten et al. [13] investigated 2D image reflection produced in a semitransparent mirror. This technique provides an optically stable 2D reflection image without auxiliary tracking, requiring only a simple preoperative alignment. As the majority of needle placement procedures are executed "in-plane" (i.e. when the needle is completely contained in a single CT slice), giving up 3D rendering for engineering simplicity and low cost appears to be a reasonable tradeoff.

This paper describes an embodiment of the 2D image overlay concept optimized for needle placement procedures in conventional CT scanners. In particular, we report new hard- 


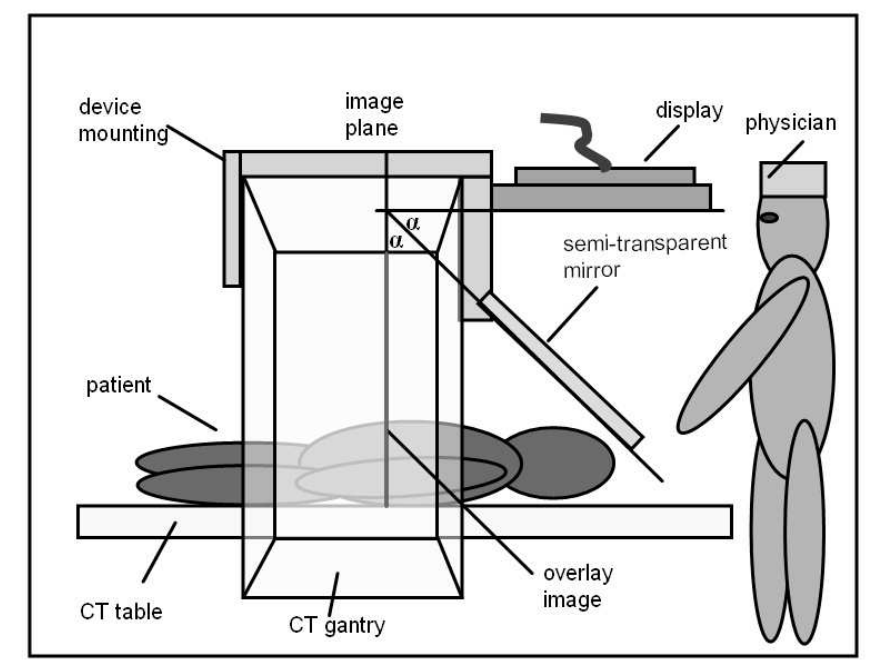

Fig. 1. System Concept

ware and display software as well as results of phantom and cadaver studies. Institutional Review Board (IRB) approval is being sought to begin human trials.

The structure of this paper is the following. After this introduction, Section 2 describes the concept of the system, and Section 3 presents the image overlay display and Section 4 the system integration. Section 5 is devoted to experiments and results, and the last section discusses the results.

\section{SYSTEM CONCEPT}

The basic concept of the two-dimensional CT image overlay is shown in Figure 1. A flat-panel display and transparent mirror are mounted on the gantry of a CT scanner. We acquire a CT slice, flip it horizontally, adjust its magnification, and then render it on the display. The scanner, display, and mirror are aligned so that the reflection of the CT image appearing in the mirror coincides with the patient's body behind the mirror as shown in Figure 2. The system creates the impression as if the CT image that was floating inside the body in the correct pose and magnification, giving the physician a planar X-ray vision. This technique can also be characterized as an in-situ visualization tool, where the medical image is rendered right in the context of the procedure, spatially registered with the physical body. Perhaps the most promising aspect of image overlay is that the physician can execute the procedure without turning his/her attention away from the patient, while executing the same series of motions and actions as in conventional freehand procedures. In most needle placement procedures, after the entry point is selected, three degrees-of-freedom (DOF) motion of the needle needs to be controlled. In this case, the physician uses the overlay image to control the in-plane insertion angle (1st DOF), while holding the needle in the axial plane marked by the gantry's inner laser light (2nd DOF). (Most modern CT scanners are equipped with two parallel laser planes: the inner plane coincides with the scanner's plane of imaging, while the outer laser plane emanates from a source mounted on the housing of the gantry over the table. The offset between the two laser planes is known from the manufacturer's specification and can also be measured by moving a marker with the encoded table between the two laser planes.) The insertion depth (3rd DOF) is controlled with a ruler and virtual depth gauge drawn on the overlay image. In addition, we may use stripes on the needle to indicate depth and a spring-loaded sterile clamp to prevent overdriving the needle (Figure 2). The use of 2D image overlay does not alter the traditional unassisted procedure to which physicians are accustomed, while increasing the amount of visual information in the field of action. The advantages of 2D image overlay are numerous in comparison to other virtual reality or display augmentation methods reviewed in the previous section. Most significantly, 2D image overlay provides optically stable image without auxiliary tracking instrumentation and it requires only a simple alignment that does not need to be repeated for each patient. The system is simple and inexpensive; the replication of the prototype system shown in (Figure 4) costs only about $\$ 3,500$. As the majority of needle placement procedures are executed "inplane" (i.e. when the needle is completely contained in a single image slice), giving up full 3D rendering for engineering simplicity and low cost appears to be acceptable. The main added benefit of the image overlay is making pre-insertion CT images available for intra-procedural guidance, without exposing the patient and physician to additional radiation. Although CT does not provide real-time imaging, the image overlay system assists the physician in detecting target motion and allows for gating the needle insertion by the respiratory cycle with the use of skin fiducials, as we explain later.

\section{CT Image Overlay Device}

We have built two basic prototypes of the CT image overlay device for pre-clinical evaluation on phantoms and human cadavers. Both prototypes are mounted on the CT gantry and rotate with the gantry if a tilted CT plane is needed to guide the needle placement. The first prototype, shown in Figure 3, produced the tomographic overlay image in the imaging plane of the scanner, inside the gantry of a singleslice CT scanner (GE Medical Systems, Waukesha, WI). The mounting mechanism was made of 2"x4" pinewood beams, providing great deal of freedom in design. To fit any position and direction of the image slice, we built a 7-DOF passive arm. The adjustable joints enabled us to adjust the angle and distance between the mirror, display, and CT gantry. As the overlay image was created in the actual imaging plane of the scanner, we achieved rapid update of the overlay image.

The second-generation prototype, shown in Figure 4, produces the reflection image in the outer axial laser plane of a Somatom-4 Siemens scanner. This arrangement provides ample room for both patient and physician. The patient must be translated in and out between imaging and intervention, which is the case in most unassisted traditional interventions anyway. No time is lost with this action, because the operator can move the table while the physician is entering the room after acquiring the targeting image. The patient is secured to the table with foam wedges and wide velcro bands, which efficiently prevent involuntary motion of the body. In all 


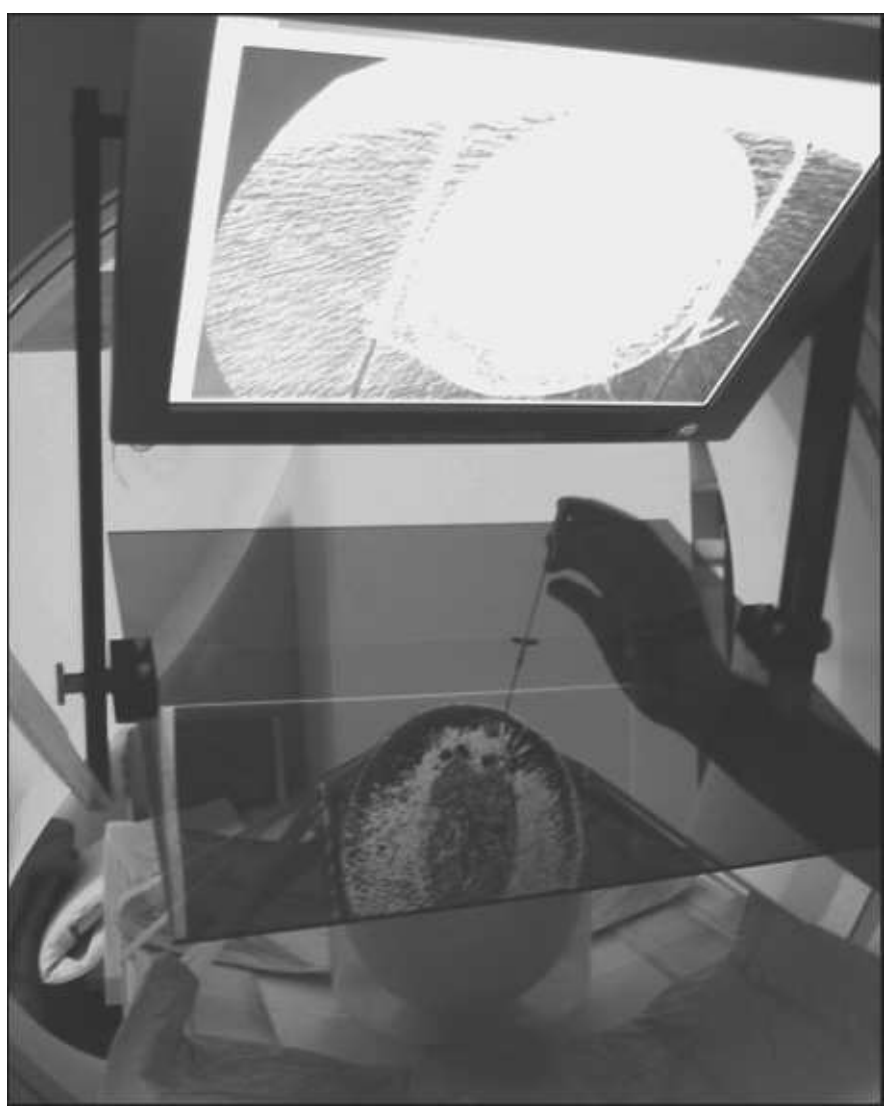

Fig. 2. Needle insertion in a head-phantom. The CT image of the water melon is floating inside the phantom.

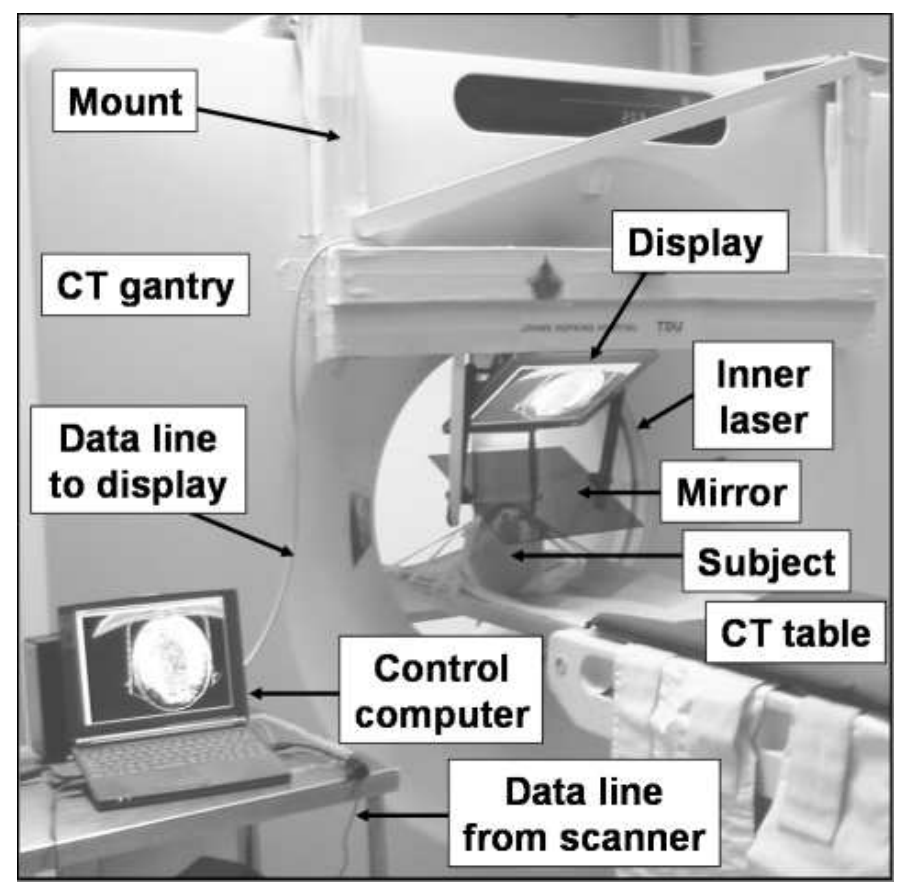

Fig. 3. First prototype of the image overlay system, the reflection image is in the inner axial laser plane of the scanner. A magnification of the system is shown in Figure 2

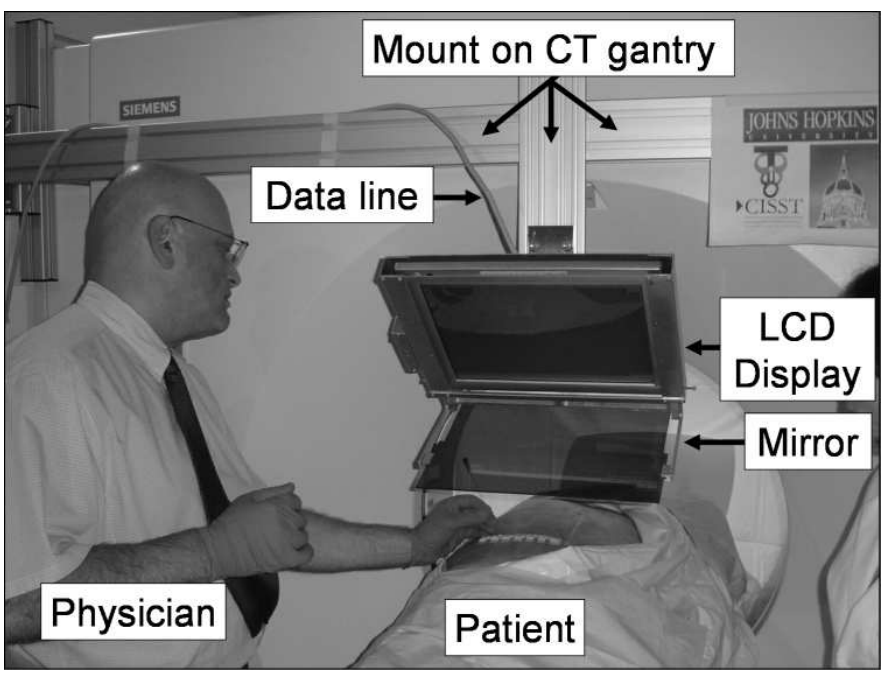

Fig. 4. Current prototype of the image overlay system, the reflection image is in the outer axial laser plane of the scanner. (The laser source is in coverage behind the mount.) Spinal needle insertion in human cadaver

modern CT scanners the table translation is digitally encoded, the linear drive mechanism is highly accurate and repeatable. Altogether, target offset due to table translation stays safely within the error margin of these procedures.

The gantry mount was built from extruded aluminum. This structure offers structural integrity, easy machining, light weight, and is aesthetically pleasing. Two average-size technicians are able to mount the basic hardware in a few minutes. The repeatability of the mount, with respect to the gantry's laser planes, is more accurate than the width of the laser (about $1.5 \mathrm{~mm}$ ), tested in ten experiments. The angle of the display and mirror are fixed with respect to one another and adjustable joints are used to set up the display-mirror unit with respect to the plane of the outer laser on the CT. As the mount is very reliable, the passive arm holding the display/mirror unit has only three adjustable joints, in contrast to the 7-DOF arm in the first prototype. The weight of the display/mirror unit and the mount is $4.0 \mathrm{~kg}$ and $20.5 \mathrm{~kg}$, respectively. This is an insignificant extra weight on the gantry and creates virtually no torque on its joints. We also verified that the overlay device did not interfere with the laser lights of the scanner.

\section{SySTEM INTEGRATION}

\section{A. Workflow}

We position the subject under the overlay on the table and we acquire a small stack of CT slices with a slice thickness appropriate for the given clinical application. IZI CT Biopsy Strip (IZI Corporation, Baltimore, MD) external fiducials are placed on the skin in the plane of interest. A single CT slice is acquired with the fiducials in place and transferred in DICOM format to the planning and control software implemented on a stand-alone computer (Dell laptop, MS Windows-XP). The laptop concurrently drives the flat panel display of the overlay device, for which the image is flipped horizontally so that the mirror would flip the image back to normal lateral polarity. We kept the insertion planning software simple and intuitive, in order to speed up the process. We refrain from 3D scenes 
altogether, which is also reasonable for a $2 \mathrm{D}$ overlay device. The CT window/level parameters can be interactively adjusted. The entry and target points are picked in the CT image by two mouse-clicks. The computer marks the target and entry points, draws a visual guide along the trajectory of insertion, and marks the depth of insertion. If the overlay system is configured in the outer laser plane of the scanner, then the table is translated out by the known offset between the slice of interest and the overlay plane. The physician holds the needle at the entry point behind the mirror and adjusts the angle to the virtual needle guide while holding the needle in the plane of the laser (Figures 2 and 4). This posture allows the physician to insert the needle with a single stroke along the marked trajectory. The IZI skin fiducials are visible on both the patient and in the overlay image. The coincidence between the corresponding marks indicates correct alignment. This feature is particularly important for quality assurance, especially in applications when the target anatomy is prone to motion due to respiration or mechanical force. The skin fiducials can be used to gate or synchronize the needle insertion to the respiratory cycle. After the needle is inserted, a confirmation image is acquired.

\section{B. Calibration}

After mounting the system, the display-mirror unit must be aligned with the scanner's plane of imaging. As we mentioned earlier, the physicians' choice was to set up the system in the outer laser plane of the scanner. (Note that the outer laser plane is parallel to the plane of imaging, the offset between the two is a priori known, and the encoded table provides precise translation between the two planes.) Calibration is accomplished in two steps: (1) make the overlay image coincide with the plane of the scanner's outer laser plane, and (2) determine the inplane transformation between the overlaid CT image and the view of the physical object in the mirror.

We fabricated a calibration phantom containing a base board and a perpendicular fiducial board, the latter with with an asymmetric set of $5 \mathrm{~mm}$ diameter and $10 \mathrm{~mm}$ long aluminum pegs, shown in Figure 5. The boards are made of $10 \mathrm{~mm}$ thick clear acrylic and they are practically radiolucent. We manually adjust the calibration phantom on the CT table until the scanner's axial laser plane sweeps the front face of the fiducial board. We render an arbitrary test image on the overlay. We adjust the joints on the mirror-display unit to make the overlay image appear on the front face of the fiducial board. This adjustment usually does not need to be performed every time the overlay device is set up, because the gantry mount is highly accurate and reproducible. (In fact, we took the mount off and then put it back ten times, and the mirrordisplay unit was correctly positioned every time and did not need any adjustment.)

The first step of the in-plane registration process is image scaling. The overlay image must appear in correct size in the mirror, but there is variable linear scaling between the CT image and displayed image. Let $\left[\begin{array}{l}u_{c} \\ v_{c}\end{array}\right]$ and $\left[\begin{array}{l}u_{d} \\ v_{d}\end{array}\right]$ be a vector in the CT image and display image, respectively, in pixel unit. Also, let $\left(p_{c x}, p_{c y}\right)$ and $\left(p_{d x}, p_{d y}\right)$ be the pixel size in the CT image and display image, respectively, in $\mathrm{mm} /$ pixel unit, in $\mathrm{x}$ and $\mathrm{y}$ directions. The pixel size of the display is constant and it is either known from the manufacturer's specification or its measurement is trivial. The pixel size of the CT image is calculated as the ratio between the field of view (in $\mathrm{mm}$ ) and image size (in pixel), both are known from the scanning protocol that is encoded in the header of the DICOM image. Then the scale factors in $\mathrm{x}$ and $\mathrm{y}$ directions are calculated as $s_{x}=p_{c x} / p_{d x}$ and $s_{y}=p_{c y} / p_{d y}$, respectively. The in-plane scaling between the CT and display image vectors is described by the following equation:

$$
\left[\begin{array}{l}
u_{d} \\
v_{d}
\end{array}\right]=\left[\begin{array}{cc}
s_{x} & 0 \\
0 & s_{y}
\end{array}\right] *\left[\begin{array}{l}
u_{c} \\
v_{c}
\end{array}\right]
$$

If the pixel size is identical in $\mathrm{x}$ and $\mathrm{y}$ directions in both the CT and display, then the scale factors are equal $\left(s_{x}=\mathrm{s}\right.$ and $\left.s_{y}=\mathrm{s}\right)$ and the equation above reduces to:

$$
\left[\begin{array}{l}
u_{d} \\
v_{d}
\end{array}\right]=\left[\begin{array}{l}
u_{c} \\
v_{c}
\end{array}\right] * s
$$

Having dealt with scaling, the second step of in-plane registration is to determine a 3-DOF rigid body transformation. We acquire a CT image of the fiducial board and render it on the overlay display, as seen in Figure 5. We adjust the in-plane rotation and translation of the phantom's CT image until each aluminum peg coincides with its mark in the CT image. We auto-segment the marks of the aluminum pegs in the phantom's CT image. Then a technician leans over the mirror and with the mouse clicks on the points where the actual pegs appear through the mirror. From the two planar sets of corresponding points, we calculate the in-plane translation and rotation. Finally, we apply the resulting 3-DOF transformation on the CT image, and then redisplay the image on the overlay. There are two possible approaches to this point-based rigid body registration: (1) Optimize the registration for minimum error in one step after collecting all corresponding pairs of points. (2) Progress gradually in an iterative cycle, where in each iteration pick only one corresponding pair and recover the translation or rotation alternately until correct registration is achieved. From our perspective, the one-step method is less practical, because using all points is usually superfluous, but using less than all of them is a gamble: if the registration needs only a tiny bit of adjustment at the end, then we still must repeat the entire registration process from the beginning. We must add, however, that the iterative registration relies on accurate point pairs, and the translation estimation in particular may be sensitive to noise (i.e. human error) and is less robust than least-squares minimization over all pairs. A severe outlier may throw off the registration, which the operator must detected visually and compensate by either undoing the outlier or adding more registration points. All facts considered, we chose the iterative rigid body registration approach, for its inherent practicality and convenience. The mathematical formulation is described next. In the equations below $\left[\begin{array}{l}u_{i} \\ v_{i}\end{array}\right]$ is a point in the CT image, $\left[\begin{array}{c}u_{i}^{\prime} \\ v_{i}^{\prime}\end{array}\right]$ is its corresponding point in 


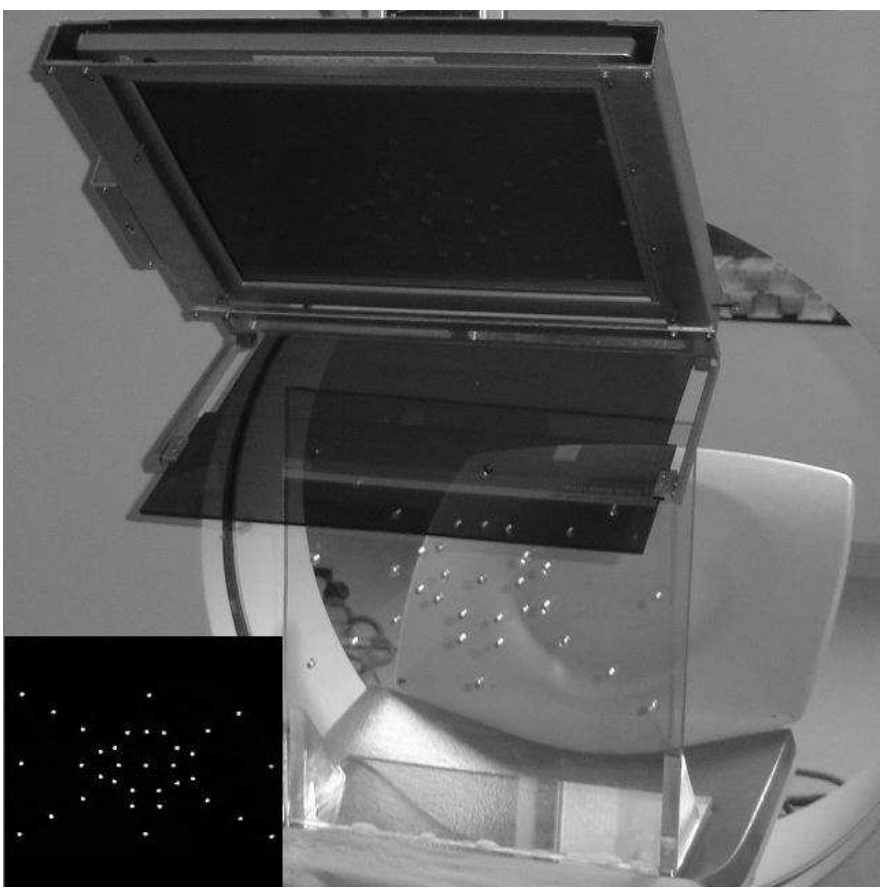

Fig. 5. Calibration phantom and its CT image at the lower left corner.

the mirror after applying the rotation or translation, $\left[\begin{array}{c}\bar{u} \\ \bar{v}\end{array}\right]$ is the center of rotation, and $\left[\begin{array}{l}p_{x} \\ p_{y}\end{array}\right]$ is the calculated translation vector.

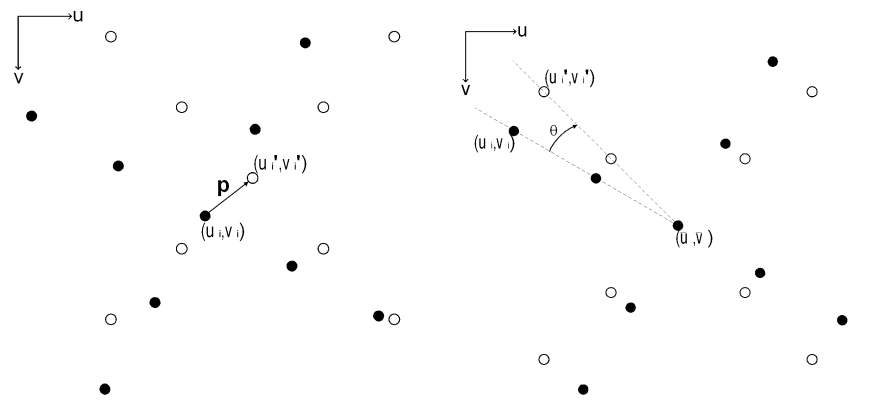

Fig. 6. In-plane rigid translation (a) and rotation (b). The aluminum pegs segmented in CT are represented by solid black dots, while the actual pegs seen in the mirror are represented by hollow circles.

We iterate in the following cycle until the exit criteria are met:

- Choose a point in the CT image, and then choose the corresponding point as seen through the mirror. (To start, pick a point near the center of the image.)

- Calculate the required translation $p$ to align the selected point, as shown in Figure 6 (a).

- Translate all CT image points by the following equation:

$$
\left[\begin{array}{c}
u_{i}^{\prime} \\
v_{i}^{\prime}
\end{array}\right]=\left[\begin{array}{l}
p_{x} \\
p_{y}
\end{array}\right]+\left[\begin{array}{l}
u_{i} \\
v_{i}
\end{array}\right]
$$

- Display the adjusted image.

- Choose another point in the CT image, and then choose the corresponding point as seen through the mirror.
- Calculate the angle $\theta$ representing the angular misalignment in the axis normal to the imaging plane. The center of rotation is the point selected previously for translation, as explained in Figure 6 (b).

- Rotate all CT points by the calculated amount about the chosen center of rotation, by the following equation:

$$
\left[\begin{array}{c}
u_{i}^{\prime} \\
v_{i}^{\prime}
\end{array}\right]=\left[\begin{array}{cc}
\cos \theta & \sin \theta \\
-\sin \theta & \cos \theta
\end{array}\right] *\left[\begin{array}{l}
u_{i}-\bar{u} \\
v_{i}-\bar{v}
\end{array}\right]+\left[\begin{array}{c}
\bar{u} \\
\bar{v}
\end{array}\right]
$$

- Display the adjusted image. (Note that the pixels in the adjusted image are interpolated, as it is always the case in rotated images.)

- Make a quick visual assessment of the registration. Continue the cycle if necessary, otherwise exit. (Note that one could also apply quantitative exit criteria on the amount translation and rotation, but we found the quick visual check to be sufficient for our clinical applications.)

We also analyzed the combined effects of human and optical errors on synthetic data, with the objective to determine the optimal number, distribution, and selection order of registration points. We artificially misaligned the two sets of points (i.e. the pegs and their respective image coordinates) by applying a known transformation on each pair and adding noise to each data point, as explained in Figure 7(a). Let $Q$ and $R$ be the corresponding points to register in CT and real space. To simulate the error induced by the user and the physical property of the semi-transparent glass, we added some "noise" to the position of $R$, obtaining point $P$. We registered $P$ to $Q$ getting a rigid-body transformation $T_{r e g}$. Using this transformation, we transformed $R$ obtaining $S$. Therefore, the system error was the distance between points $S$ and $Q$. Human error was simulated by adding a random value (2 pixels) to the position of the point to be registered. Parallax error from the mirror may induce a significantly large error. At $\mathrm{d}=4 \mathrm{~mm}$ thickness and $\alpha=30^{\circ}$ view angle and using the formulas derived in Figure 7(b), the parallax error was about $x=1.28 \mathrm{~mm}$, the effect of which fortunately can be substantially reduced by altering the view points during the calibration process. We also concluded that iterative re-registration was more favorable than collecting all registration points first and then calculating a single global registration. This observation was true across the range of practically relevant amplitudes and distributions of noise and number of registration points. We found the following to be the best strategy: (1) Pick a peg near the area of interest in the vicinity of expected target and entry. Let the computer calculate translation and readjust the overlay image. (2) Pick a peg far out from the previous one. Let the computer calculate rotation and readjust the overlay image. (3) Pick a new peg again relatively close to area of interest to adjust translation. (4) Pick another peg to adjust rotation, far out and in $90^{\circ}$ from the previous one. (5) Repeat the pairwise process until sufficient registration is observed between the phantom and its CT image. It is particularly important to compensate for the parallax error by altering the viewpoint. The registration error, using four pairs of registration points and assuming 2 pixel human error was $1.23 \mathrm{~mm}(\mathrm{STD}=0.31)$ at $30^{\circ}$ view angle and $0.74 \mathrm{~mm}(\mathrm{STD}=0.21)$ at perpendicular 


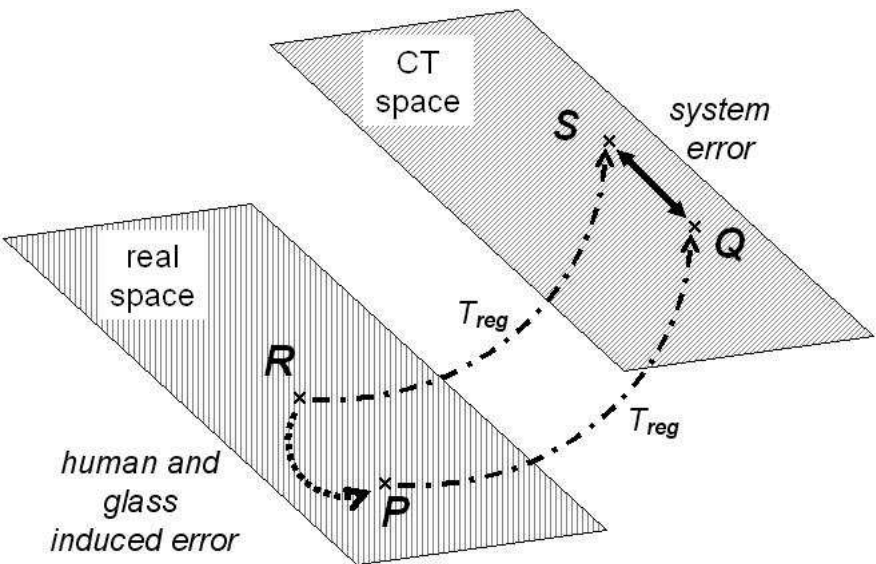

(a)

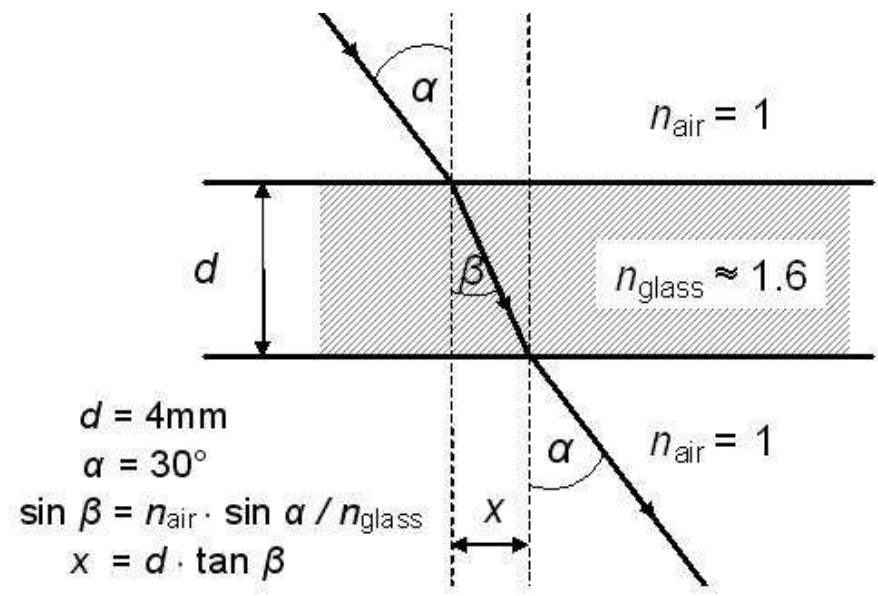

(b)

Fig. 7. Definition of registration error (a) and the calculation of parallax error (b).

view $\left(\alpha=0^{\circ}\right)$. This performance appears to be adequate for most CT-guided needle placement procedures.

\section{EXPERIMENTS AND RESULTS}

\section{A. Head Phantom}

In this experiment we used the prototype presented in Figure 3. The overlay device was set up in the plane of imaging, so the subject was not moved between imaging and needle placement. We implanted a honeydew melon with multiple $1.5 \mathrm{~mm}$ diameter metal pellets serving as targets. The melon simulated the locations of head, neck, and intracranial targets, as seen in Figure 2. We used standard brain imaging protocol with $1 \mathrm{~mm}$ slice thickness, and standard $20 \mathrm{G}$ diamond-head stainless steel needles. The needles came with a sterilized spring loaded clamp that was adjusted to the depth of insertion. An experienced interventionalist executed three interventions. We assessed the accuracy in post-insertion CT, with $1 \mathrm{~mm}$ slice thickness. The needle tip approached the implanted metal ball target within $2 \mathrm{~mm}$ distance in every attempt. Although the metal ball was not distinguishable from the needle in the confirmation CT slice, the lateral scout view clearly showed the target and needle tip. The needle was fully visible in $\mathrm{CT}$ slice, indicating that the physician succeeded in holding the needle exactly in the laser plane.

\section{B. Body Phantoms}

The overlay device, mounted in the inner laser plane of the scanner, demonstrated several ergonomic shortcomings: (1) the physician's workspace was often restricted, (2) in some situations the target was inaccessible, (3) the subject could not be translated in and out the scanner without moving the mirror out of the way, (4) the gantry could not be tilted beyond a few degrees without collision between the subject and mirror, (5) in some cases there was not enough room for the needle between the subject and mirror. Due to the limitations identified during the head phantom experiments, the unanimous decision of the participating clinicians was to configure the device in the outer laser plane of the scanner. In all subsequent experiments, this system configuration was used. Although the subject needs to be moved between imaging and needle insertion, that was not considered as a significant risk in addition to the standard procedure. There is some time delay after taking the targeting image, because the interventionalist must return to the room. Furthermore, the IZI Biopsy Strip fiducials provide sensitive external indication of body motion, thereby mitigating the risk of target dislocation substantially.

We constructed a male body phantom by attaching three cm-thick tissue-equivalent bolus material (Harpell Associates Inc., Oakville, ON, Canada) on the back of a plastic male torso and placing $1.5 \mathrm{~mm}$ diameter metal balls in the bolus at various depths. This phantom represented a male upper body in prone position, with hard fat/muscle layer with mechanical targets in the back, spine, and shoulder. The objectives were to demonstrate accurate needle placement at the pre-marked targets and assess the ergonomics of the system. An experienced interventionalist executed four needle insertions with $18 \mathrm{G}$ diamond needles. We assessed the accuracy of needle placement in post-insertion CT, with $1 \mathrm{~mm}$ slice thickness. The needle approached the implanted target within $2 \mathrm{~mm}$ in every attempt. There was no interference in the workspace and all targets were conveniently accessible. The ergonomics of the system was also tested on a commercial interventional phantom (CIRS, Norfolk, VA), resulting in the same outcome. Also importantly, the reproduction error of the CT gantry mount relative to the CT laser was consistently below $2 \mathrm{~mm}$ (which is less than the width of the laser), tested in 10 trials.

\section{Cadaver Experiments}

The objective of human cadaver experiments was to demonstrate the ability of clinically successful needle placement to various anatomical targets in several potential clinical applications. The accuracy of needle placement was assessed in post-insertion CT. The slice thickness was 1.0-3.0 mm, which was clinically adequate for the procedures in our study.

1) Spinal Nerve Blocks and Facet Joint Injections: are demanding procedures primarily because of the sheer volume of cases treated regularly by the neurological pain management 


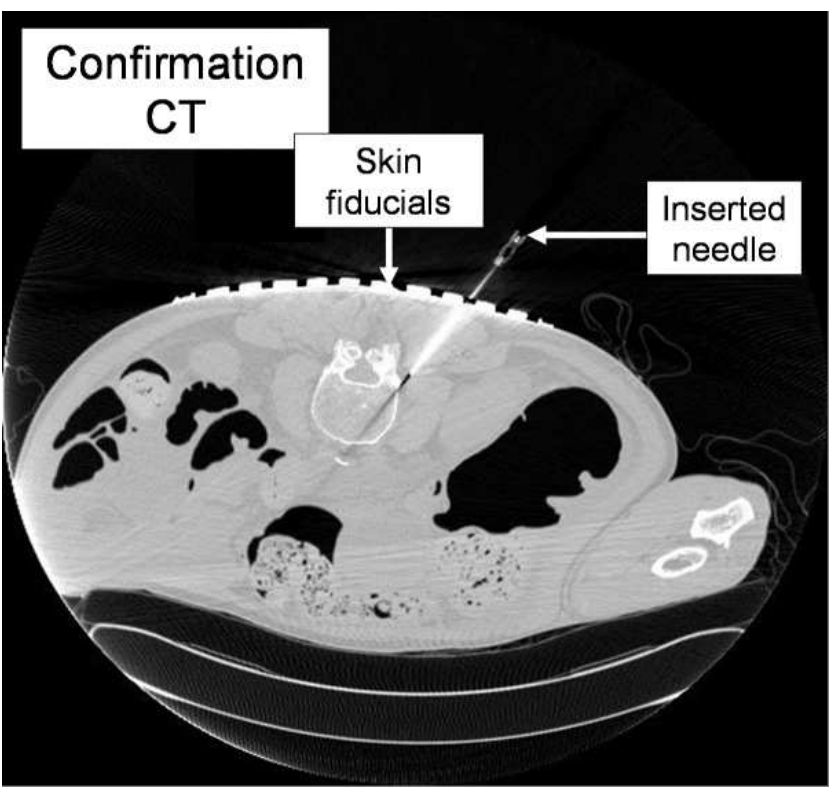

Fig. 8. CT of confirmation of needle placement for spinal pain management

service at our institution. The interventional team is under strong pressure to perform these procedures; namely bilateral injections under 10 minutes and with clinically sufficient placement accuracy of about $2.0 \mathrm{~mm}$.

In our experiments, a cadaver was set up in prone position for nerve root and facet joint injections in the lumbar spine area. We performed several needle insertions with $22 \mathrm{G}$ beveled needles at the L4 and L5. Contrast and therapeutic substances were not injected. All four spinal nerve block and facet joint needle placements were clinically successful. A confirmation CT image is shown in Figure 8, where the needle tip touches the correct anatomical target. Here needle is completely included in the confirmation image, indicating the physician managed to keep the needle exactly in the gantry's laser plane.

2) Shoulder and Hip Arthrographies: are diagnostic procedures frequently applied for assessment of joint injury. An Magnetic Resonance Imaging (MRI) contrast agent is injected percutaneously into the injured joint under CT or Xray fluoroscopy guidance and then the patient is brought to an MRI imaging facility for diagnostic scanning.

In our experiments, we fixed a female cadaver in supine position and performed four needle insertions to the shoulder and hip joint, using $22 \mathrm{G}$ beveled needles. The accuracy of needle placement was assessed in CT and then contrast substance was also injected in two cases. All four needle placements for joint arthrography of the shoulder and hip were clinically successful as the injection needle safely entered the joint space, two of those cases are shown in Figure 9. Each needle enters the body in the selected entry point, proceeds along the marked trajectory, and touches the selected anatomical target point in the joint space. Contrast material (not shown in the figures) was very hard to inject into the stiffened joint of the cadaver and it did not distribute as evenly as it does in live patients.

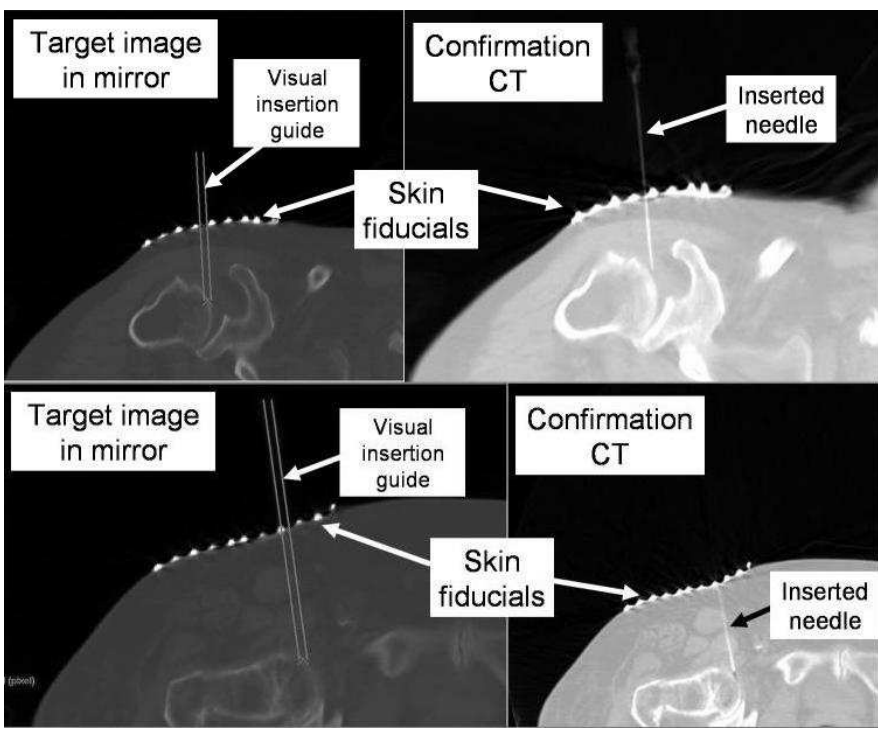

Fig. 9. Target and confirmation CT for arthrography of shoulder (top) and hip joint (bottom)

3) Pilot Needle Insertion for Musculoskeletal Biopsy: Typically, performance of a CT guided percutaneous musculoskeletal (MSK) biopsy requires careful attention to target and path planning, followed by insertion of a $22 \mathrm{G}$ pilot needle to the bone surface through the pre-selected entry point and path. After correct placement of the pilot needle is ascertained with a confirmatory CT image, a large (12$16 \mathrm{G})$ or smaller (14-18 G) core needle can be passed down along the pilot needle to collect the sample from a skeletal or soft tissue lesion respectively. Bone biopsies are especially demanding procedures because the needle must make contact with the bone in some predefined angle that is often very difficult to achieve freehand. One must also avoid sensitive structures (like nerves and blood vessels) in the path. As we almost always operate on damaged bone, faulty placement and misalignment of the large needle or drill may cause structural damage to the already weakened bone; in fact, fracture is not an insignificant intra-operative risk. Many patients have preexisting micro-fractures, a condition that may develop into a full fracture if the instrument is accidentally wedged in the fracture line. Altogether, unassisted freehand planning of the skin entry, trajectory, bone entry, and cortical target can become exceedingly difficult.

In our experiments, we fixed a female cadaver in supine position and performed four needle insertions to the pelvis, using $22 \mathrm{G}$ beveled needles. A $1.0 \mathrm{~mm}$ steel ball was implanted percutaneously into the right lateral wall of the left pelvis, and then this location was targeted from several directions from different entry points. All four pilot needle insertions for pelvic bone biopsy were clinically successful, the confirmation CT is shown in Figure 10. Unfortunately, the artifact caused by the needles suppresses the signal of the implanted metal target, but the needles converge in a confined area, indicating appropriate accuracy for bone biopsy. Three of the four needles are completely included in the image slice, while the fourth one is slightly deflected from the image plane. 


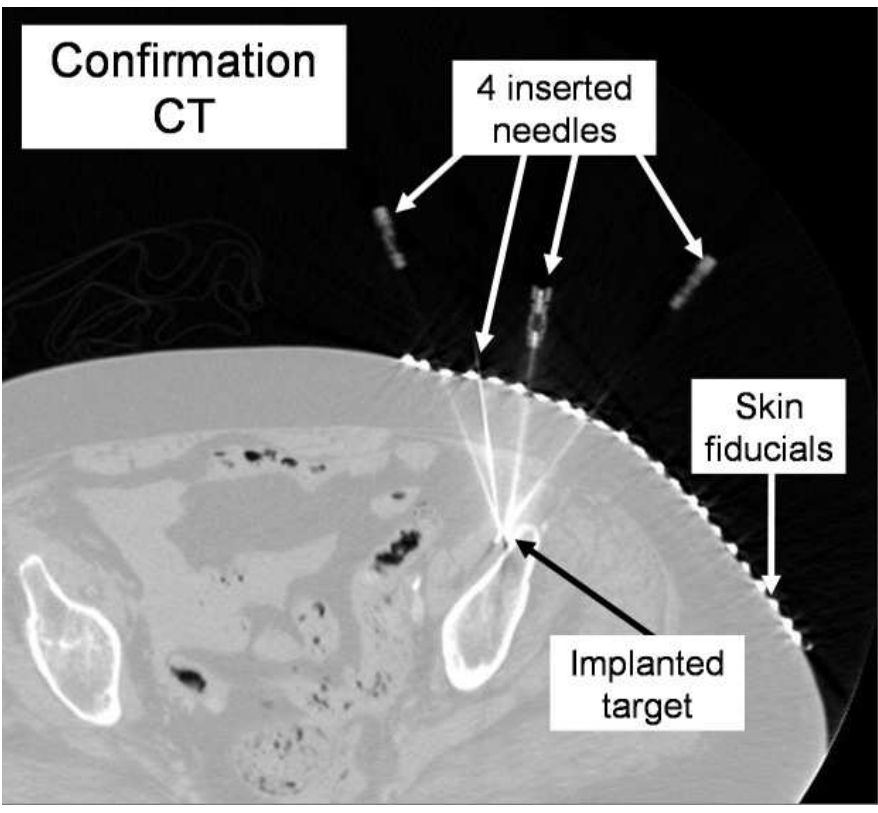

Fig. 10. Confirmation CT of pilot needle insertion in bone biopsy.

\section{DISCUSSION}

We have reviewed alternative augmented realty solutions earlier in Section 1. Here we provide a critical analysis of our results with the CT image overlay device. Overall, phantom and cadaver experiments were successful toward proving the hypothesis: the CT image overlay device allowed for clinically adequate and safe needle placement without the need for reinsertion and re-imaging. As our efforts now are gearing up for clinical trials, several ergonomic and technical issues need to be dealt with.

In our first prototype (see Figure 3) the image overlay device was mounted in the inner laser plane of the scanner. The physician's workspace was often restricted and several targets were not accessible. Therefore, the decision of the participating physicians was to use the outer laser plane of the scanner (see Figure 4), even though it required translation of the CT table between imaging and needle insertion. (This does not cause time delay, because the physician needs time to enter the scanner room anyway.)

Initially, optical parallax used to cause problems during needle insertion. Our current workflow, however, eliminates that effect: We identify the needle insertion point on the patient's skin with the use of the IZI biosy strip markers, rather than picking the point in the overlay image. Once the needle tip is committed, we keep the needle parallel to the virtual needle guide drawn on the overlay image. Parallax can make the needle appear shifted with respect to the virtual guide. We know, however, that this is just an optical illusion, because the needle is firmly lodged in its entry point. We simply disregard the parallax shift and concentrate on keeping the needle parallel to the virtual guide.

Needle insertion was more accurate in phantoms than in human cadavers, for several reasons. First, the phantom tissue was softer and more homogeneous than human cadavers. Also, in phantoms we used $18 \mathrm{G}$ diamond tip needles that barely deflected during insertion, while in the human cadavers we used $22 \mathrm{G}$ beveled needles that had a stronger tendency to deflect in the hard and inhomogeneous tissues. There are several methods to mitigate deflection during free-hand needle insertion, such as spinning or various combinations of jackhammering, spinning, and insertion. It must also be noted that thin needles are mostly used in relatively superficial targets, so the off-axis dislocation of the needle tip usually remains under the clinical margin of error. One must keep in mind that needle bending is not always unwanted. For example, beveled needles are often purposely deflected off some bony structures to access narrow passages, as often is the case in facet joint and epidural space injections. Furthermore, needle bending does not necessarily cause the needle tip to land outside the clinical margin of error, however, pinpoint accuracy is always more desirable. Finally, we reiterate that the main goal of our project is not pinpoint accuracy, but the prevention of clinically faulty needle insertions.

Although respiration could not be simulated in the passive phantoms and cadavers, the IZI Biopsy Strip fiducials placed on the patient around the insertion point are expected to provide robust real-time indication of any patient motion, in which case the insertion can be halted till the patient's body is correctly re-registered. The IZI fiducials can be used for reregistration of the patient in the same manner as the calibration fixture is registered. Importantly, this can be accomplished without re-imaging the patient.

Despite continuous improvement, calibration of the system still is a significant issue. Optical parallax and human perception errors need to be eliminated from the process. Note that the prototype in Figure 4 uses the outer laser plane on the CT gantry. Aligning the display/mirror unit with this laser plane is a difficult and also somewhat unsafe practice, because the laser source is mounted on the cover of the gantry rather than on its steel frame and thus the accuracy of the beam cannot be guaranteed. A more appealing solution is to construct a rigid display/mirror unit that carries its own laser reference plane, similarly to Stetten [13] and calibrate the system to the scanner as described in [8]. This device will be aligned to the CT scan plane with two rotational joints, while the remaining four degrees of freedom will be compensated by software. The calibration of this system will be fully automated and free of human perception errors. These modifications are currently underway in our laboratory.

We have also encountered several other minor problems. The physician sometimes had difficulty in holding the needle in the axial laser plane. Although this presently does not seem to be a clinically significant impediment, we consider applying some form of soft mechanical constraint to aid the physician with holding the needle in plane. We have found it somewhat inconvenient to have the planning laptop in the scanner room, especially because its wire connection occasionally disrupted normal traffic. In the forthcoming clinical version, we will place the laptop next to the console and apply wireless communication to the device in the scanner room. Changes in room light conditions have also caused difficulties. Although we draped the distal opening of the gantry, foreground light still often interfered with the overlay image. We consider 
mounting a controlled light source on the frame and reduce occasional glare from the display frame by applying dark satin coating.

In summary, needle placement experiments with both phantoms and cadavers were clinically successful and confirmed in post-insertion CT imaging. The workflow process and instrumentation have not been optimized to yield minimum procedure time and the ergonomics of the system still need to improve; these are subjects to current research. Nevertheless, the current embodiment was found to be adequate and safe for initial human trials in several of needle placement applications, in which the previously described cadaver studies are predictive of good clinical performance. Presently, Institutional Review Board (IRB) approval is being sought to commence human trials in MSK biopsy and arthrography while software and hardware refinements will continue.

\section{ACKNOWLEDGMENT}

Funding was available from NSF EEC-9731478, Siemens Corporate Research (Princeton, NJ), and Japanese Ministry of Education, Science, Sports and Culture, Grant-in-Aid for Young Scientists \#14702071. We are grateful to Beatrice Mudge, RTT and Frank Sauer, PhD (Siemens) for help and advice.

\section{REFERENCES}

[1] H. Birkfellner, W. Figl, M. Huber, K. Watzinger, F. Wanschitz, F. Hummel, J. Hanel, R. Greimel, W. Homolka, P. Ewers, and R. Bergmann. A head-mounted operating binocular for augmented reality visualization in medicine - design and initial evaluation. IEEE Transactions on Medical Imaging, 21(8):991-997, 2002.

[2] M. Blackwell, C. Nikou, A.M. DiGioia, and T. Kanade. An image overlay system for medical data visualization. In First International Conference on Medical Image Computing and Computer-Assisted Intervention (MICCAI), Lecture Notes in Computer Science 1496, pages 232-240, 1998.

[3] K. Cleary and C. Nguyen. State of the art in surgical robotics: clinical applications and technology challenges. Comput Aided Surgery, 6(6):312-328, 2001

[4] DiGioia et al. Computer aided surgery. Cybersurgery, pages 121-139, 1998.

[5] W.E.L. Grimson, G.J. Ettinger, S.J. White, L. Lozano-Perez, W.M. Wells III, and R Kikinis. An auto-matic registration method for frameless stereotaxy, image guided surgery, and enhanced reality visualization. IEEE Transactions on Medical Imaging, 15(2):129-139, 1996.

[6] H. Iseki, Y. Masutani, M. Iwahara, T. Tanikawa, Y. Muragaki, T. Taira, $\mathrm{T}$ Dohi, and K. Takakura. Volumegraph (overlaid three-dimensional image-guided navigation). clinical application of augmented reality in neurosurgery. Stereotact Funct Neurosurg, 68:18-24, 1997.

[7] R.A. Kockro, L. Serra, Y. Tseng-Tsai, C. Chan, S. Yih-Yian, C. GimGuan, E. Lee, L.Y. Hoe, N. Hern, and W.L. Nowinski. Planning and simulation of neurosurgery in a virtual reality environment. Neurosurgery, 46(1):118-135, 2000.

[8] S. Lee, G. Fichtinger, and G.S. Chirikjian. Novel algorithms for robust registration of fiducials in CT and MRI. Journal of Medical Physics, 29(8):1881-1891, 2002.

[9] K. Masamune, G. Fichtinger, A. Deguet, D. Matsuka, and R.H. Taylor. An image overlay system with enhanced reality for percutaneous therapy performed inside ct scanner. In Fifth International Conference on Medical Image Computing and Computer-Assisted Intervention (MICCAI), Lecture Notes in Computer Science 2488, pages 77-84, 2002.

[10] K. Masamune, Y. Masutani, S. Nakajima, I. Sakuma, T. Dohi, H. Iseki, and K. Takakura. Three-dimensional slice image overlay system with accurate depth perception for surgery. In Third International Conference on Medical Image Computing and Computer-Assisted Intervention (MICCAI), Lecture Notes in Computer Science 1935, pages 395-402, 2000.
[11] S. Nakajima, K. Nakamura, K. Masamune, I. Sakuma, and T. Dohi. Three-dimensional medical imaging display with computer-generated integral photography. Computerized Medical Imaging and Graphics, 25:235-241, 2001.

[12] F. Sauer, A Khamene, and S Vogt. An augmented reality navigation system with a single-camera tracker: System design and needle biopsy phantom trial. In Fifth International Conference on Medical Image Computing and Computer-Assisted Intervention (MICCAI), Cambridge England, Lecture Notes in Computer Science 2489, pages 116-124, 2002.

[13] G.D. Stetten and V.S. Chib. Overlaying ultrasonographic images on direct vision. J Ultrasound Med., 20(3):235-240, 2001.

[14] R.H. Taylor and D. Stoianovici. Medical robotics in computer-integrated surgery. IEEE Transaction of Robotics and Automation, 19(5):765-781, 2003.

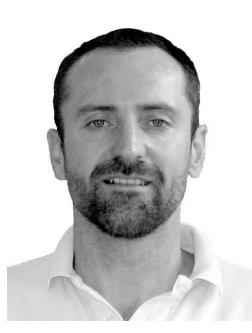

Gabor Fichtinger (M04) received the BS and MS degrees in Electrical Engineering, and the PhD degree Computer Science from the Technical University of Budapest, Hungary, in 1986, 1988, and 1990, respectively. He completed a post-doctoral training in high performance computing at the University of Texas at Austin, with a specialization in biomedical visualization and computing. From 1993 to 1999 , he lead the development of radiosurgery, radiotherapy, and neurosurgery treatment planning systems at the George Washington University and Precision Mathematics, Inc. Currently, Dr. Fichtinger is an Associate Research Professor of Computer Science, Mechanical Engineering and Radiology at the Johns Hopkins University. His research focuses on image-guided surgical interventions, surgical planning, medical robotics, surgical navigation, therapy monitoring, and systems integration.

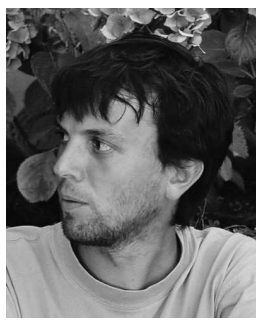

Anton Deguet received the BS in Mathematics in 1991 from the University of Tours and the MS in computer science in 1994 from the University of Grenoble, France. Since 2000, he has been a research systems engineer at the Center for Computer Integrated Surgery at the Johns Hopkins University.

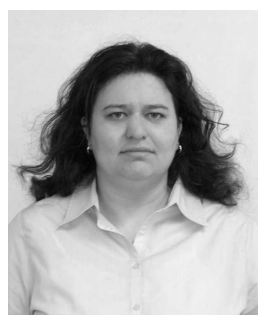

Emese Balogh received the BS/MS combined degree in computer science in 1995 from the BabesBolyai University in Cluj, Romania and completed doctoral requirements in computer science in the University of Szeged, Hungary. Since 2003, she has been at the Johns Hopkins University as a predoctoral research fellow. Emese Balogh's research interest is in the area of medical image processing, computer graphics, discrete tomography, and image guided needle insertions. 


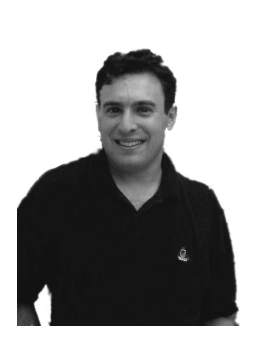

Gregory S. Fischer received the BS degrees of Mechanical Engineering and Electrical Engineering from Rensselaer Polytechnic Institute, Troy, NY in 2002 and the MSE degree in Electrical Engineering from The Johns Hopkins University, Baltimore, MD in 2004. Since 2002, he has been a graduate research assistant at the Center for Computer Integrated Surgery at The Johns Hopkins University, and is currently working towards the $\mathrm{PhD}$ degree in Mechanical Engineering.

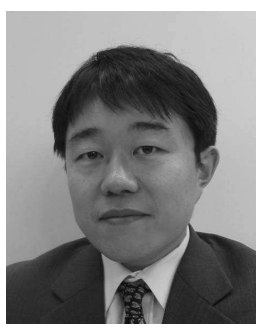

Ken Masamune received the BS, MS, and $\mathrm{PhD}$ degrees in precision machinery engineering from the University of Tokyo in 1993,1995, and 1999, respectively. He is the recipient of several research awards from the Japan Society of Computer Aided Surgery and the Japan Society of Medical Electronics and Biological Engineering. Currently, he is an Associate Professor at the Department of Intelligent and Mechanical Engineering, Tokyo Denki University, Japan. Dr. Masamune's research interests span over all aspects of computer assisted surgery, with speacial regard to MRI-compatible mechatronic manipulators.

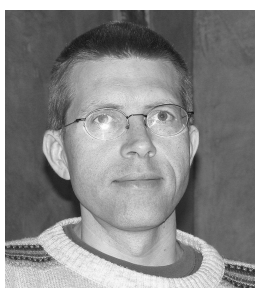

Herve Mathieu received the engineering degree in 1988 from the cole Centrale Lille, France. In 2002, he was a research engineer at the Center for Computer Integrated Surgery at the Johns Hopkins University. He worked on robotics systems used in computer aided surgery and radiology. Currently, he is research engineer at the French National Institute for Research in Computer Science and Control (INRIA). Herve Mathieu's expertise concerns hardware and software integration in Computer Vision and Robotics domains.

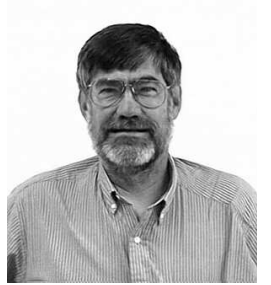

Russell H. Taylor (M76F94) received the B.E.S. degree from The Johns Hopkins University, Baltimore, MD in 1970 and the Ph.D. degree in computer science from Stanford University, Stanford, CA in 1976. He joined IBM Research in 1976, and in September 1995, he moved to Johns Hopkins University as a Professor of Computer Science, with joint appointments in Radiology and Mechanical Engineering. He is also Director of the NSF Engineering Research Center for Computer-Integrated Surgical Systems and Technology. In 19881989, he led the team that developed the first prototype for the ROBODOC system for robotic hip replacement surgery. At IBM, he subsequently developed novel systems for computer-assisted craniofacial surgery and roboticallyaugmented endoscopic surgery. At Johns Hopkins, he has worked on all aspects of computer-assisted surgery, including modelling, registration, and robotics in areas including percutaneous local therapy, microsurgery, and computer-assisted bone cancer surgery. Dr. Taylor is Editor Emeritus of the IEEE TRANSACTIONS ON ROBOTICS AND AUTOMATION, a Fellow of the AIMBE, and a member of various honorary societies, panels, editorial boards, and program committees. In February 2000, he received the Maurice Mller award for excellence in computer-assisted orthopedic surgery.

S. James Zinreich received BS degree from the University of Maryland and MD degree from the University of Brussels. He completed residency in Sinai and the Johns Hopkins University. Currently,

PLACE PHOTO HERE he is a professor of radiology and head and neck surgery at the Johns Hopkins University. Over the past two decades, Dr. Zinreich has been a premier innovator in image-guided neurosurgery and endoscopic sinus surgery and served as advisor to several major imaging companies.

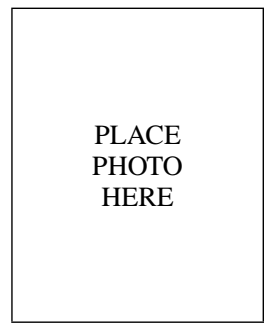

Laura M. Fayad received the BS and MS degrees in mechanical engineering from MIT and Stanford University, respectively and the MD degree from McGill University Faculty of Medicine. Currently, she is an assistant professor of radiology at the Johns Hopkins University. Her clinical practice and basic research includes image-guided biopsy and other diagnostic procedures of the musculoskeletal system. 